\title{
Multiobjective Image Color Quantization Algorithm Based on Self-Adaptive Hybrid Differential Evolution
}

\author{
Zhongbo Hu, ${ }^{1}$ Qinghua Su, ${ }^{1}$ and Xuewen $\mathrm{Xia}^{2}$ \\ ${ }^{1}$ School of Information and Mathematics, Yangtze University, Jingzhou, Hubei 434023, China \\ ${ }^{2}$ School of Software, East China Jiaotong University, Nanchang 330013, China \\ Correspondence should be addressed to Qinghua Su; suqhdd@126.com
}

Received 19 July 2016; Revised 24 August 2016; Accepted 4 September 2016

Academic Editor: Manuel Graña

Copyright (c) 2016 Zhongbo Hu et al. This is an open access article distributed under the Creative Commons Attribution License, which permits unrestricted use, distribution, and reproduction in any medium, provided the original work is properly cited.

In recent years, some researchers considered image color quantization as a single-objective problem and applied heuristic algorithms to solve it. This paper establishes a multiobjective image color quantization model with intracluster distance and intercluster separation as its objectives. Inspired by a multipopulation idea, a multiobjective image color quantization algorithm based on self-adaptive hybrid differential evolution (MoDE-CIQ) is then proposed to solve this model. Two numerical experiments on four common test images are conducted to analyze the effectiveness and competitiveness of the multiobjective model and the proposed algorithm.

\section{Introduction}

Image color quantization is one of the common image processing techniques. It is the process of reducing the number of colors presented in a color image with less distortion [1]. Most of the image color quantization methods [2-12] are essentially based on data clustering algorithms. Recently, some heuristic methods, such as genetic algorithm (GA) [13, 14], particle swarm optimization algorithm (PSO) [15-17], and differential evolution (DE) [18-21], have been employed to solve the image color quantization problems which are considered as optimization problems. Evaluation criteria, which are used as objective functions of optimization problems, often incorporate mean square-error (MSE) [22-24], intracluster distance $\left(\bar{d}_{\max }\right)$, and intercluster separation $\left(d_{\min }\right)[25-28]$.

Most of the image color quantization algorithms based on heuristic methods are single-objective methods; that is, only one evaluation criterion is used. References [26-28] have used three evaluation criteria, but their three criteria have been merged to get a linear weighting objective function. In general, the objective function in any of the above algorithms holds only one evaluation criterion or a linear combination of several evaluation criteria. This paper presents the following two aspects:

(i) Develop multiobjective model for image color quantization problems. Based on the model, we can obtain a quantized image with the smallest color distortion among those images which meet a trade-off between the optimal color gradation and the optimal color details.

(ii) Propose a multiobjective algorithm based on a selfadaptive DE for solving the multiobjective image color quantization model.

The rest of the paper is organized as follows. Section 2 establishes a multiobjective image color quantization model. Section 3 presents a multiobjective image color quantization algorithm based on self-adaptive hybrid DE (MoDE-CIQ). Experimental results and discussion on four test images are provided in Section 4. Conclusions are given in Section 5.

\section{Establishment of a Multiobjective Image Color Quantization Model}

2.1. Multiobjective Image Color Quantization Model. In single-objective models, mean square-error (MSE) (1) is the 
most popular evaluation criterion for color image quantization [29]. Intracluster distance $\left(\bar{d}_{\max }\right)(2)$ and intercluster separation $\left(d_{\text {min }}\right)(3)$ come next in importance to MSE. Smaller MSE means smaller color distortion. Smaller $\bar{d}_{\max }$ means smoother gradation of similar colors. Larger $d_{\min }$ means more color details to be preserved. The three evaluation criteria are expressed in the following formulas [28]:

$$
\begin{aligned}
& \text { MSE }=\frac{1}{M \times N} \sum_{i=1}^{M} \sum_{j=1}^{N} \min _{k \in\{1,2, \ldots, K\}}\left\{d\left(I(i, j), c_{k}\right)\right\} \\
& \bar{d}_{\max }=\max _{k=1,2, \ldots, K}\left\{\frac{\left[\sum_{\forall I_{p} \in C_{k}} d\left(I_{p}, c_{k}\right)\right]}{\left|C_{k}\right|}\right\}, \\
& d_{\text {min }}=\min _{\forall k_{1}, k_{2}=1,2, \ldots, K, k_{1} \neq k_{2}} d\left(c_{k_{1}}, c_{k_{2}}\right) .
\end{aligned}
$$

Here, $M \times N$ is the size of a color image $I . I(\cdot, \cdot)$ is a pixel in $I$. $K$ is a given color number of a colormap. $k$ is the sequence number of the colors in the colormap. $c_{k}$ is the $k$ th color of the colormap. $k_{1}$ and $k_{2}$ are two different sequence numbers of the colors in the colormap. $C_{k}$ is the cluster of all pixels in $I$ with similar color to $c_{k}$. $\left|C_{k}\right|$ is the number of all pixels in $C_{k} . I_{p}$ is the color of a pixel in $C_{k} \cdot d(\cdot, \cdot)$ represents Euclidean distance.

This paper proposes a multiobjective image color quantization model which uses two evaluation criteria, $\bar{d}_{\max }$ and $d_{\text {min }}$, as its subobjective functions. The model can be formulized as follows:

$$
\begin{aligned}
\operatorname{minimize} & F(x)=\left(g_{1}(x), g_{2}(x)\right)^{T} \\
\text { s.t. } & x \in[0,255]^{3 \times K} .
\end{aligned}
$$

Here $[0,255]^{3 \times K}$ is decision space. Decision vector $x$ is a colormap consisting of $K$ randomly selected color triples in the color space $[0,255]^{3}$. Let

$$
c_{k}=\left(x_{3 k-2}, x_{3 k-1}, x_{3 k}\right), \quad k=1,2, \ldots, K,
$$

be the $k$ th color of the colormap. Then

$$
\begin{aligned}
x & =\left(c_{1}, c_{2}, \ldots, c_{K}\right) \\
& =\left(x_{1}, x_{2}, x_{3}, x_{4}, x_{5}, x_{6}, \ldots, x_{3 K-2}, x_{3 K-1}, x_{3 K}\right) .
\end{aligned}
$$

$F(x)$ is the objective function with the following two subobjectives:

$$
\begin{aligned}
& g_{1}(x)=\bar{d}_{\max }, \\
& g_{2}(x)=255-d_{\min } .
\end{aligned}
$$

This model aims to make a trade-off between $\bar{d}_{\max }$ minimum and $d_{\min }$ maximum. The solution set of this multiobjective model is called Pareto set, the solutions of which could balance color gradation and color details.
Obviously, the solution with the smallest MSE in the Pareto set of the above multiobjective model corresponds to a quantized image, which holds the smallest color distortion among those images with a balance between the optimal color gradation and the optimal color details.

2.2. Conflict Detection of the Subobjective Functions. As we all know, the subobjective functions of a multiobjective model should be conflicting. This means, as two subobjectives in the above model, $g_{1}(x)$ and $g_{2}(x)$ should not become better simultaneously. Namely, when $\bar{d}_{\max }$ becomes better (smaller), $d_{\min }$ should not also become better (larger). In this part, several experiments are conducted to show that the subobjective functions, $g_{1}(x)$ and $g_{2}(x)$, in the above model are obviously conflicting.

Figure 1 shows four common test images (Peppers, Baboon, Lena, and Airplane) with size $512 \times 512$ pixels. Reference [15] presented a color image quantization algorithm based on self-adaptive hybrid DE (SaDE-CIQ), in which the objective function is MSE. We, respectively, replace its objective with $\bar{d}_{\max }$ and $d_{\min }$ to obtain two algorithms, named SaDE-CIQ1 and SaDE-CIQ2. SaDE-CIQ, SaDE-CIQ1, and SaDE-CIQ2 are implemented to quantize all test images into the quantized images with 16 colors. Each algorithm is run 10 times on each test image. In the three algorithms, there are two parameters, a maximum iteration $t_{\max }$ and a mixed probability $p$. Here, $t_{\max }=200$. For showing the same relation of MSE, $\bar{d}_{\max }$ and $d_{\min }$ for the different values of $p$, we let $p$ take three different values, $0.1,0.05$, and 0.01 in the three algorithms.

For the three algorithms with different $p$, we can get the similar relation of MSE, $\bar{d}_{\max }$ and $d_{\min }$. So, we only use the part results of SaDE-CIQ1 with $p=0.1$ as an example to analyze the relation of MSE, $\bar{d}_{\max }$ and $d_{\min }$. By any image and its quantized image, we can calculate the values of MSE, $\bar{d}_{\max }$ and $d_{\min }$. Table 1 gives all the objective values $\bar{d}_{\max }$ of SaDECIQ1 in 10 runs and the corresponding values of MSE and $d_{\text {min }}$. Figure 2 shows the changes of these values in 10 runs. We include the curves of Peppers from first run to second run as an example of how to illustrate the conflicts of MSE, $\bar{d}_{\max }$ and $d_{\min }$. When $\bar{d}_{\max }$ becomes better (smaller), $d_{\min }$ does not become better (larger). When MSE becomes better (smaller), $d_{\text {min }}$ does not become better (larger). When $\bar{d}_{\max }$ becomes better (smaller), MSE also becomes better (smaller). These mean $\bar{d}_{\max }$ and $d_{\min }$ are conflicting, MSE and $d_{\min }$ are conflicting, and $\bar{d}_{\max }$ and MSE are not conflicting. According to the statistical analysis for all test images, there are 15 conflicts between $\bar{d}_{\max }$ and $d_{\min }, 16$ between MSE and $d_{\min }$, and 11 between $\bar{d}_{\max }$ and MSE. These statistical data show that any two of MSE, $\bar{d}_{\max }$ and $d_{\min }$, are in conflict.

In summary, for the conflict of $\bar{d}_{\max }$ and $d_{\min }$, it is appropriate to select them as the subobjective functions in the above multiobjective image color model. Meanwhile, for the conflicts of MSE with $\bar{d}_{\max }$ and $d_{\min }$, there does not exist preference when MSE is applied to select the solution in the Pareto set of the above multiobjective model. 
TABLE 1: The results of 10 runs for SaDE-CIQ1 $(p=0.1)$.

\begin{tabular}{|c|c|c|c|c|c|c|c|c|c|c|c|}
\hline \multirow{2}{*}{ Test image } & & \multicolumn{10}{|c|}{ Test serial number } \\
\hline & & 1 & 2 & 3 & 4 & 5 & 6 & 7 & 8 & 9 & 10 \\
\hline \multirow{3}{*}{ Peppers } & $\bar{d}_{\max }$ & 27.8885 & 26.9858 & 26.0681 & 28.1934 & 25.7472 & 32.8054 & 26.8729 & 32.0317 & 25.5597 & 28.0979 \\
\hline & $d_{\min }$ & 41.5508 & 29.3541 & 25.0297 & 37.4886 & 40.54 & 33.6902 & 35.1127 & 36.4135 & 38.3825 & 36.1761 \\
\hline & MSE & 26.8221 & 26.2667 & 25.1661 & 26.8304 & 25.5318 & 31.5104 & 24.1938 & 29.6623 & 23.7019 & 27.8777 \\
\hline \multirow{3}{*}{ Baboon } & $\bar{d}_{\max }$ & 26.6224 & 27.6260 & 28.3537 & 26.8452 & 26.6689 & 30.5386 & 27.6907 & 28.4376 & 26.8122 & 28.7434 \\
\hline & $d_{\min }$ & 36.2255 & 32.8375 & 34.9105 & 24.7064 & 36.5621 & 30.9652 & 26.8745 & 25.9984 & 33.4011 & 38.2255 \\
\hline & MSE & 20.3766 & 20.8404 & 20.1511 & 19.6093 & 20.9290 & 19.4481 & 21.6021 & 20.2362 & 19.4163 & 20.5033 \\
\hline \multirow{3}{*}{ Lena } & $\bar{d}_{\max }$ & 27.2745 & 34.0579 & 28.5068 & 26.6540 & 26.6780 & 37.2558 & 12.6332 & 34.9201 & 28.0219 & 33.1166 \\
\hline & $d_{\min }$ & 21.9009 & 36.3725 & 33.3204 & 37.1832 & 37.1524 & 26.1205 & 37.3509 & 28.9622 & 24.5176 & 29.3508 \\
\hline & MSE & 8.3868 & 15.5077 & 28.0535 & 5.6724 & 15.9792 & 40.6224 & 9.5826 & 38.4419 & 17.6261 & 13.4949 \\
\hline \multirow{3}{*}{ Airplane } & $\bar{d}_{\max }$ & 21.9009 & 36.3725 & 33.3204 & 37.1832 & 37.1524 & 26.1205 & 37.3509 & 28.9622 & 24.5176 & 29.3508 \\
\hline & $d_{\min }$ & 8.3868 & 15.5077 & 28.0535 & 5.6724 & 15.9792 & 40.6224 & 9.5826 & 38.4419 & 17.6261 & 13.4949 \\
\hline & MSE & 15.5626 & 25.9173 & 26.8238 & 22.2865 & 26.5673 & 20.2143 & 29.551 & 25.9917 & 21.0685 & 24.9274 \\
\hline
\end{tabular}

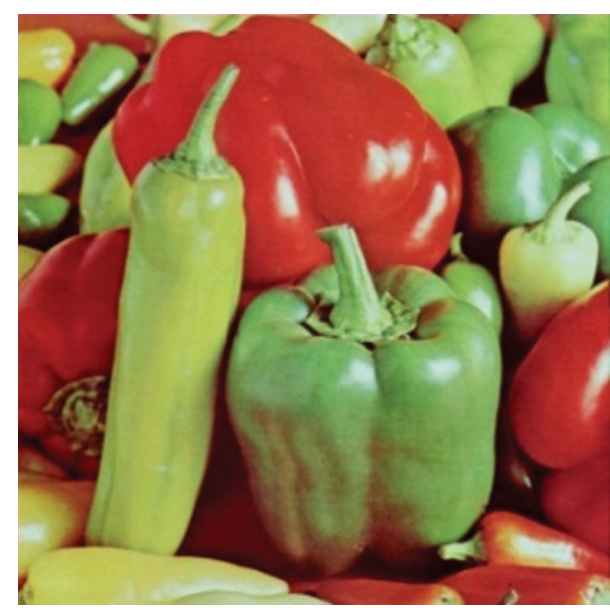

(a) Peppers

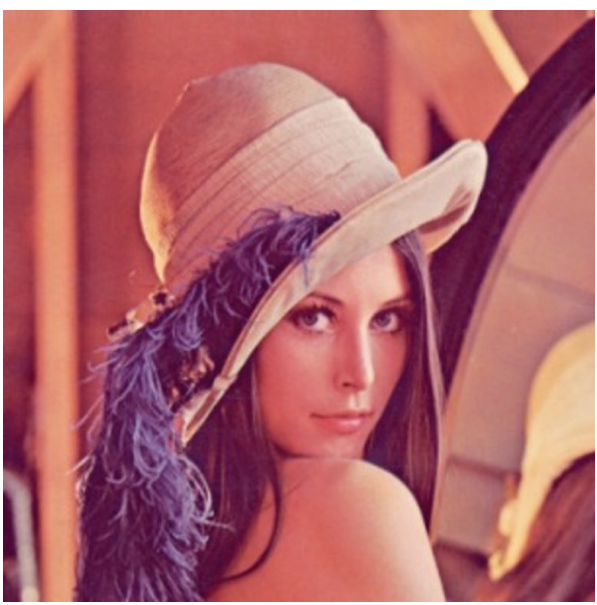

(c) Lena

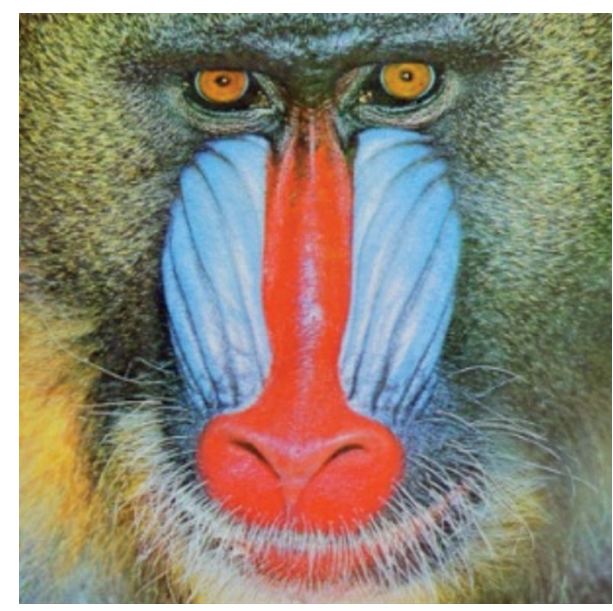

(b) Baboon

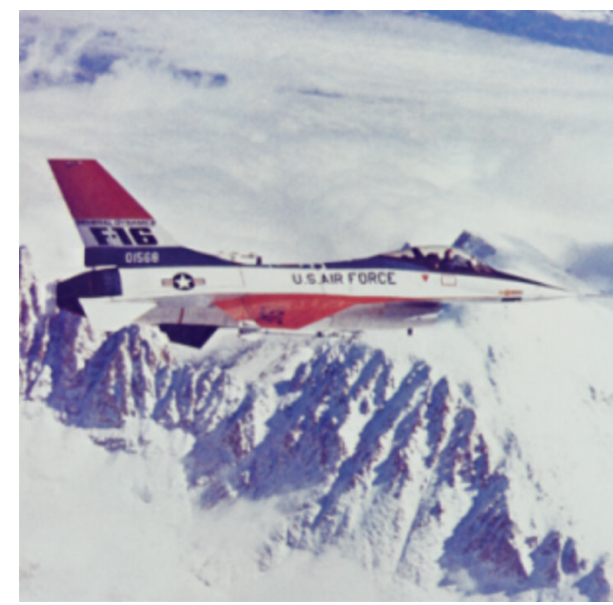

(d) Airplane

Figure 1: Test images. 

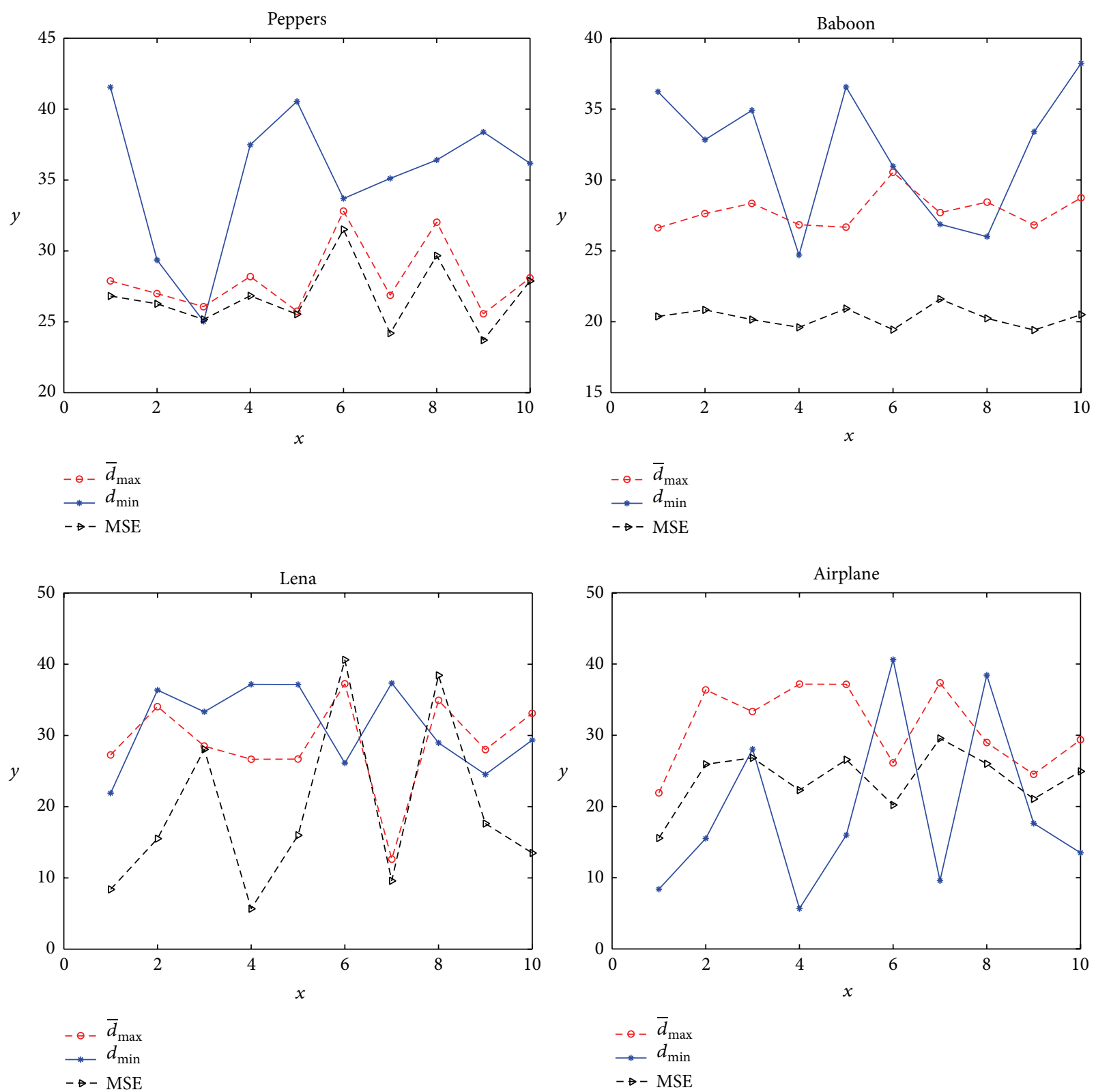

FIGURE 2: The curves of $\bar{d}_{\max }, d_{\min }$, and MSE obtained by SaDE-CIQ1 $(p=0.1)$.

\section{Multiobjective Image Color Quantization Algorithm Based on Self-Adaptive Hybrid DE}

For solving the above multiobjective image color quantization model, this section proposes a multiobjective image color quantization algorithm based on self-adaptive hybrid DE (MoDE-CIQ). This algorithm merges the ideas of SaDECIQ in [19] and a multipopulation DE algorithm VEDE [30], which is a Pareto-based multiobjective DE algorithm. The main steps of the proposed MoDE-CIQ algorithm are described as below.
Step 1 (initialize populations). Two initial populations including NP individuals are randomly selected separately. Here, each individual is a colormap with $K$ colors from an image $I$. The initial populations are denoted by

$$
\begin{aligned}
& X_{1}=\left\{x_{1}, x_{2}, \ldots, x_{\mathrm{NP}}\right\}, \\
& X_{2}=\left\{x_{\mathrm{NP}+1}, x_{\mathrm{NP}+2}, \ldots, x_{2 * \mathrm{NP}}\right\} .
\end{aligned}
$$

Step 2 (optimize populations). The population $X_{1}$ is updated by SaDE-CIQ with $g_{1}(x)$ as its objective. The population $X_{2}$ is updated by SaDE-CIQ with $g_{2}(x)$ as its objective. Then, the best individuals of the two populations are exchanged. The update and exchange operations are repeated to achieve 


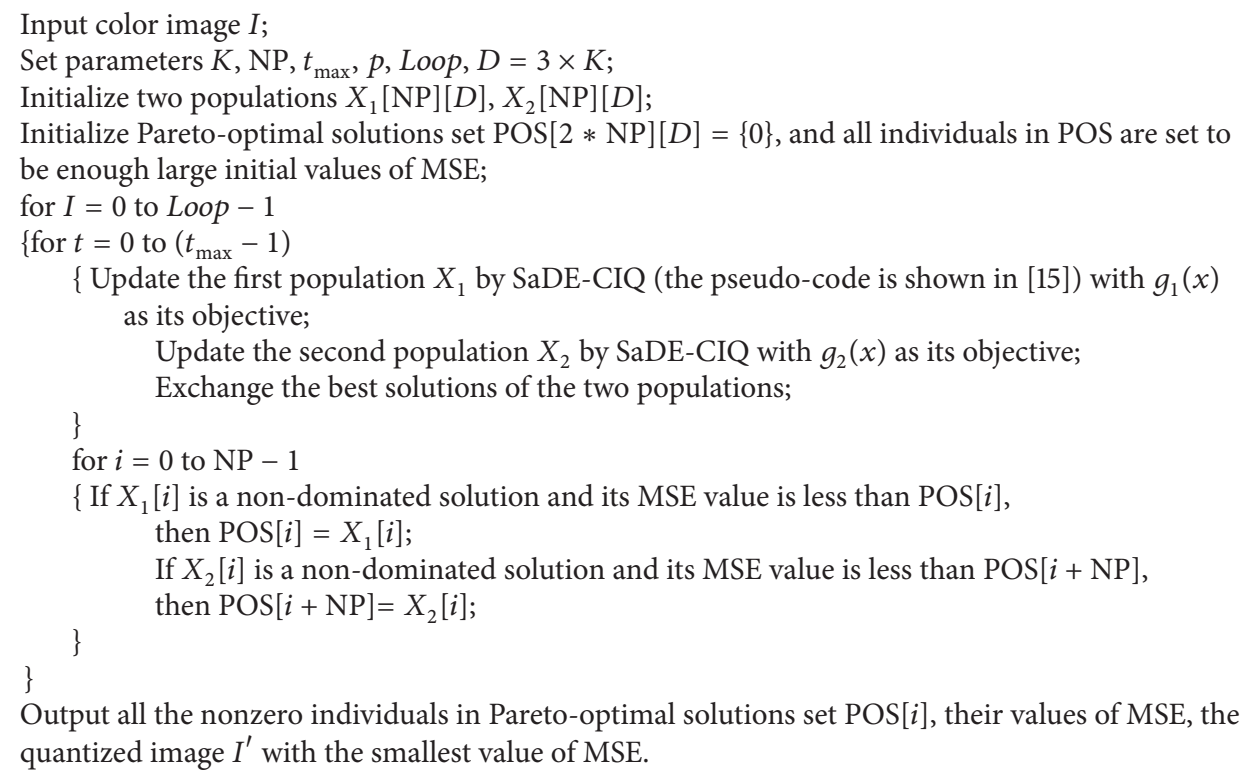

Pseudocode 1: The pseudocode of MoDE-CIQ.

TABLE 2: The MSE values resulting from SaDE-CIQ, $K$-means, and PSO-CIQ.

\begin{tabular}{lccccccc}
\hline \multirow{2}{*}{ Alg. } & \multicolumn{2}{c}{ Peppers } & \multicolumn{2}{c}{ Baboon } & \multicolumn{2}{c}{ Lena } & \multicolumn{2}{c}{ Airplane } \\
& Min & Max & Min & Max & Min & Max & Min \\
\hline SaDE-CIQ & 17.4682 & 18.7266 & 22.7496 & 23.3382 & 12.9709 & 13.8055 & 8.2482 \\
K-means & 18.1086 & 21.2676 & 22.9532 & 24.9563 & 15.6401 & 19.1314 & 9.1141 \\
PSO-CIQ & 36.3436 & 40.9532 & 35.8892 & 41.9940 & 29.6644 & 34.5867 & 21.3540 \\
\hline
\end{tabular}

a predetermined iteration number $t_{\max }$. The set of $t_{\max }$ th generation individuals of the two populations is denoted by

$$
X=\left\{x_{1}^{t_{\max }}, x_{2}^{t_{\max }}, \ldots, x_{\mathrm{NP}}^{t_{\max }}, x_{\mathrm{NP}+1}^{t_{\max }}, x_{\mathrm{NP}+2}^{t_{\max }}, \ldots, x_{2 * \mathrm{NP}}^{t_{\max }}\right\}
$$

Step 3 (reserve nondominated solutions). All nondominated solutions in $X$ are recorded in a set POS.

(Note: for an individual $x_{i}^{t_{\max }}(i=1,2, \ldots, 2 * \mathrm{NP})$, if there is no another one $x_{j}^{t_{\max }}(j \neq i, j=1,2, \ldots, 2 * \mathrm{NP})$ such that $g_{1}\left(x_{j}^{t_{\max }}\right)<g_{1}\left(x_{i}^{t_{\max }}\right)$ and $g_{2}\left(x_{j}^{t_{\max }}\right)<g_{2}\left(x_{i}^{t_{\max }}\right)$, that is, $F\left(x_{j}^{t_{\max }}\right) \prec F\left(x_{i}^{t_{\max }}\right)$, it is a nondominated solution. Otherwise, it is a dominated solution.)

Step 4 (obtain an approximative Pareto solution set). Steps 2 and 3 are repeated to achieve a predetermined iteration number Loop. The final set POS is recorded as an approximative Pareto solution set.

Step 5 (determine an optimal solution). In the set POS, the solution with the smallest values of MSE is finally reserved as an optimal solution of an image color quantization problem.

The pseudocode of MoDE-CIQ is shown as Pseudocode 1.

\section{Numerical Experiments}

In this section, two sets of experiments are conducted to illustrate the effectiveness of MoDE-CIQ algorithm and the advantage of the multiobjective model, respectively.

\subsection{Experiments for Showing the Multiobjective Algorithmic Superiority}

4.1.1. Experimental Background. Currently, the heuristic algorithms employed to solve the image color quantization problem have mainly GA, PSO, and DE. Reference [16] indicated that PSO is superior to GA. In [31], DE and PSO show similar performance on image color quantization. However, due to simple operation, litter parameters, and fast convergence, DE is the better choice to use than PSO. These mean that $\mathrm{DE}$ is a competitive image color quantization in the heuristic algorithms for image color quantization. Reference [19] proposed a color image quantization algorithm based on self-adaptive hybrid DE (SaDE-CIQ), in which the parameters of DE are automatically adjusted by a selfadaptive mechanic. Then, SaDE-CIQ is compared with $K$ means and the color image quantization algorithm using PSO (PSO-CIQ). Table 2 shows the smallest and the largest objective values for the three algorithms over 10 runs obtained 
TABLE 3: The best MSE values and the corresponding objective values of MoDE-CIQ.

\begin{tabular}{|c|c|c|c|c|}
\hline Image & $p$ values & $\bar{d}_{\max }$ & $d_{\text {min }}$ & MSE \\
\hline \multirow{3}{*}{ Peppers } & 0.1 & 25.6127 & 28.2967 & 19.1029 \\
\hline & 0.05 & 28.2967 & 31.9070 & 18.8444 \\
\hline & 0.01 & 24.8917 & 38.4062 & 19.5632 \\
\hline \multirow{3}{*}{ Baboon } & 0.1 & 27.8841 & 45.8284 & 22.9602 \\
\hline & 0.05 & 27.8083 & 45.5262 & 22.9887 \\
\hline & 0.01 & 27.9030 & 44.7175 & 22.9654 \\
\hline \multirow{3}{*}{ Lena } & 0.1 & 20.2311 & 26.4388 & 14.2847 \\
\hline & 0.05 & 20.2849 & 32.0907 & 15.6655 \\
\hline & 0.01 & 21.1913 & 32.9181 & 15.5229 \\
\hline \multirow{3}{*}{ Airplane } & 0.1 & 22.0570 & 24.1028 & 10.7517 \\
\hline & 0.05 & 22.0105 & 29.6160 & 11.2520 \\
\hline & 0.01 & 20.9759 & 26.9999 & 10.9591 \\
\hline
\end{tabular}

TABLE $4: \bar{d}_{\max }, d_{\min }$, and MSE of the quantized images with 16 colors by three algorithms.

\begin{tabular}{|c|c|c|c|c|c|}
\hline Image & $p$ values & Algorithm & $\bar{d}_{\max }$ & $d_{\min }$ & MSE \\
\hline \multirow{3}{*}{ Peppers } & \multirow{3}{*}{0.1} & MoDE-CIQ & 25.6127 & 28.2967 & 19.1029 \\
\hline & & SaDE-CIQ3 & 34.2489 & 45.8673 & 20.3563 \\
\hline & & SaDE-CIQ & 37.2450 & 22.2473 & 17.4577 \\
\hline \multirow{3}{*}{ Baboon } & \multirow{3}{*}{0.1} & MoDE-CIQ & 27.8841 & 45.8284 & 22.9602 \\
\hline & & SaDE-CIQ3 & 27.8122 & 45.8426 & 22.9592 \\
\hline & & SaDE-CIQ & 28.1805 & 36.4773 & 22.7644 \\
\hline \multirow{3}{*}{ Lena } & \multirow{3}{*}{0.1} & MoDE-CIQ & 20.2311 & 26.4388 & 14.2847 \\
\hline & & SaDE-CIQ3 & 22.8824 & 27.5461 & 13.5264 \\
\hline & & SaDE-CIQ & 22.2973 & 19.0143 & 12.9641 \\
\hline \multirow{3}{*}{ Airplane } & \multirow{3}{*}{0.05} & MoDE-CIQ & 22.0105 & 29.6160 & 11.2520 \\
\hline & & SaDE-CIQ3 & 113.2050 & 34.8630 & 17.4217 \\
\hline & & SaDE-CIQ & 23.7529 & 8.2540 & 8.0544 \\
\hline
\end{tabular}

in [19]. The results show that SaDE-CIQ is an effective color image quantization algorithm, and SaDE-CIQ has better quantization quality than $K$-means and PSO-CIQ. It is naturally to be thought that SaDE-CIQ is the best one of the image color quantization algorithms based on heuristic algorithms.

Reference [28] presented a linear weighting objective function of $\bar{d}_{\max }$ and $d_{\min }$ and MSE below:

$$
g=w_{1} \bar{d}_{\max }+w_{2}\left(255-d_{\min }\right)+w_{3} \cdot \mathrm{MSE},
$$

where $w_{1}, w_{2}$, and $w_{3}$ are the user-defined weights of the subobjectives. The linear weighting objective function (10) is the only one, including the three evaluation criteria of MoDE-CIQ, in existing references. So in this section, we will compare MoDE-CIQ, SaDE-CIQ, and SaDE-CIQ3 obtained by replacing the objective function MSE with the linear weighting objective function (10) in SaDE-CIQ.

4.1.2. Experimental Design. MoDE-CIQ, SaDE-CIQ, and SaDE-CIQ3 are implemented to quantize the four test images in Figure 1 into the quantized images with 16 colors. Each algorithm is run 10 times. The parameters of algorithms are set as follows:

$K=16, \mathrm{NP}=100, t_{\max }=200$, Loop $=5$. Mixed probability $p$ takes three different values, $0.1,0.05$, and 0.01. $w_{1}, w_{2}$, and $w_{3}$ take the same values as those in [28].

4.1.3. Experimental Results. For MoDE-CIQ, Table 3 reports the best MSE values and the corresponding objective values $\bar{d}_{\text {max }}, d_{\text {min }}$ in 10 runs. In fact, smaller $\bar{d}_{\text {max }}$ is better, larger $d_{\text {min }}$ is better, and smaller MSE is better. As shown in Table 3, the following conclusions are obtained. (i) For Peppers, only MSE is best as $p=0.05 . \bar{d}_{\max }$ and $d_{\min }$ are best as $p=0.01$. As $p=0.1, \bar{d}_{\text {max }}, d_{\text {min }}$, and MSE are all medians, and $\bar{d}_{\max }$ and MSE are similar to their corresponding best values. (ii) For Baboon, as $p=0.1, d_{\min }$ and MSE are all best. (iii) For Lena, $\bar{d}_{\max }$ and MSE are all best as $p=0.1$. (iv) For Airplane, as 


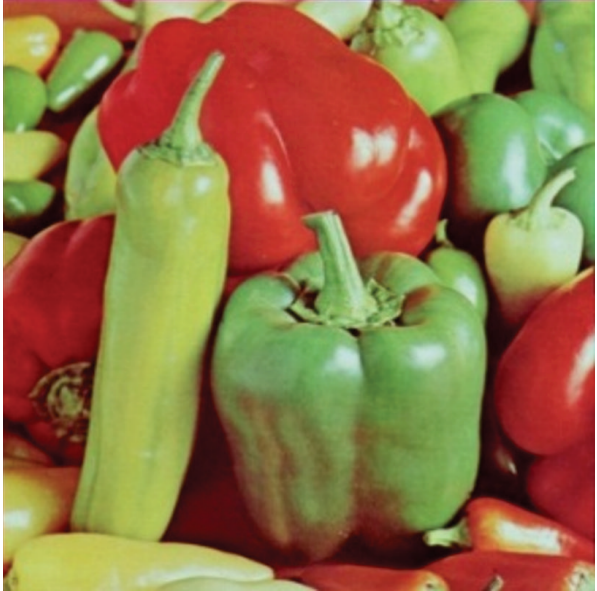

(a) Peppers

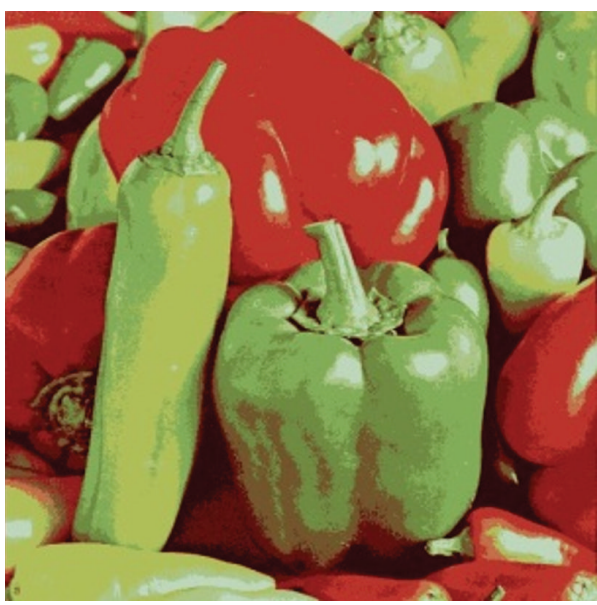

(c) SaDE-CIQ3

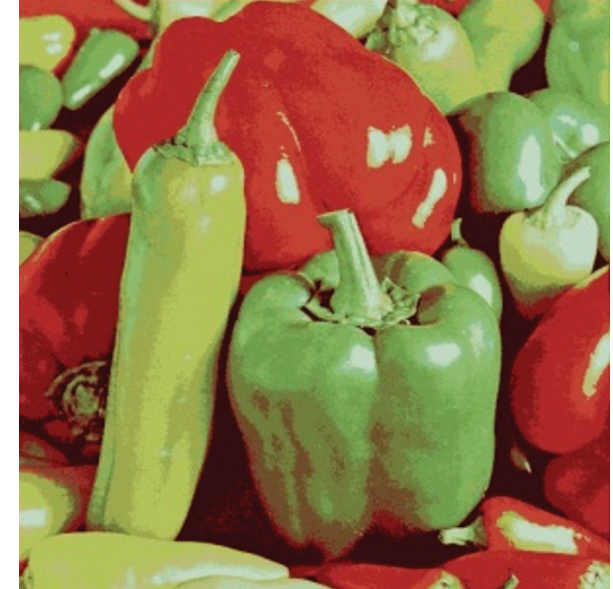

(b) MoDE-CIQ

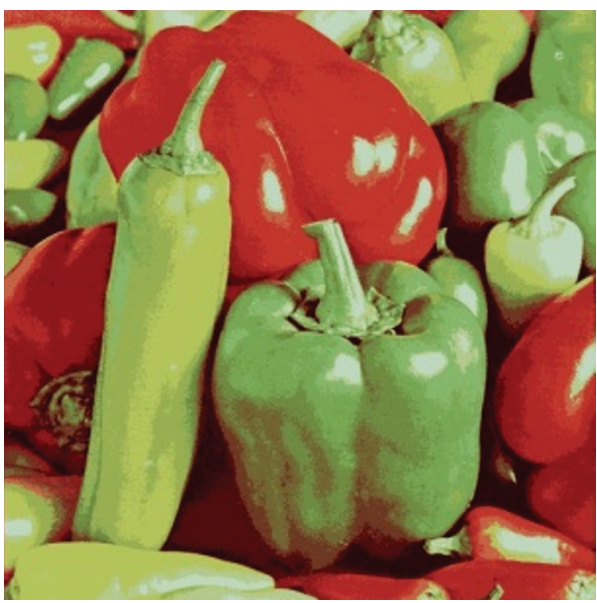

(d) SaDE-CIQ

Figure 3: The quantized images of Peppers with 16 colors obtained by three algorithms.

$p=0.05, \bar{d}_{\max }$ is best, $d_{\min }$ is a median, and MSE is similar to the other two values.

According to the above conclusions, we will take $p$ as 0.1 for Peppers, Baboon, and Lena in the following comparing experiments. However, there are few and extremely unequally distributed base colors in Airplane. For preserving main color gradations and richer color levers of original images, $\bar{d}_{\max }$ should be as small as possible. So we will take $p$ as 0.05 for Airplane in the following comparing experiments.

For comparing MoDE-CIQ, SaDE-CIQ, and SaDE-CIQ3, Table 4 reports $\bar{d}_{\max }, d_{\min }$, and MSE of their quantized images, MSE values of which are the smallest in their 10 runs. SaDE-CIQ aims to minimize its objective MSE, so its values of MSE are surely the best than those of other two algorithms. But the values of $\bar{d}_{\max }$ and $d_{\min }$ by MoDE-CIQ are all better than those of SaDE-CIQ. The values of $\bar{d}_{\max }$ and $d_{\text {min }}$ obtained by SaDE-CIQ3 for Peppers and Baboon are also better than those of SaDE-CIQ. The values of $\bar{d}_{\max }$, $d_{\text {min }}$, and MSE obtained by MoDE-CIQ are better than those of SaDE-CIQ3, except for their similar values of $\bar{d}_{\max }, d_{\min }$, and MSE for Baboon, and the values of MSE for Lena. Figures 3, 4, 5, and 6 show all quantized images of the four common test images in Figure 1. In Figures 3-6, all subfigures (a) are the original test images. Subfigures (b), subfigures (c), and subfigures (d) are the quantized images separately obtained by MoDE-CIQ, SaDE-CIQ3, and SaDE-CIQ. The visual effects of the quantized images are compared as follows. (i) For Peppers (shown in Figure 3), there are contrasting and equally distributed main base colors, so the quantized images obtained by three algorithms visually have similar color distortions. The differences in the quantization quality of these quantized images depend on their color gradations of larger regions with similar colors. The quantized images of MoDE-CIQ and SaDE-CIQ have the more rich color levers than the one of the SaDE-CIQ3. (ii) For Baboon (shown in Figure 4), there are also contrasting and equally distributed main base colors, but there are little larger regions with similar colors. So the quantized images of three methods have 


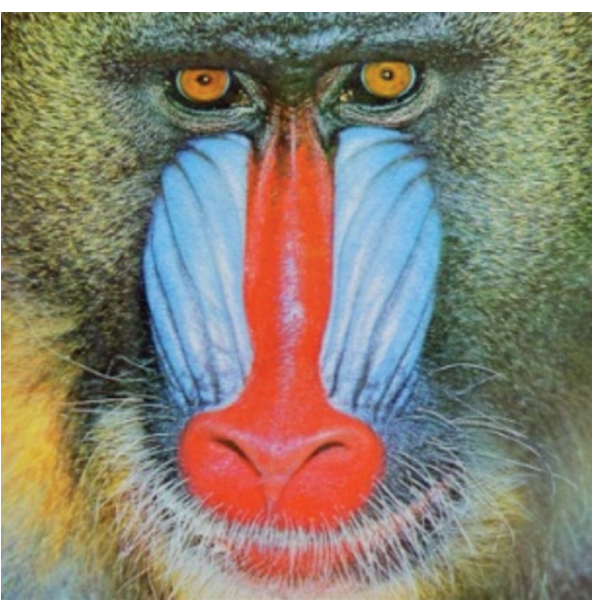

(a) Baboon

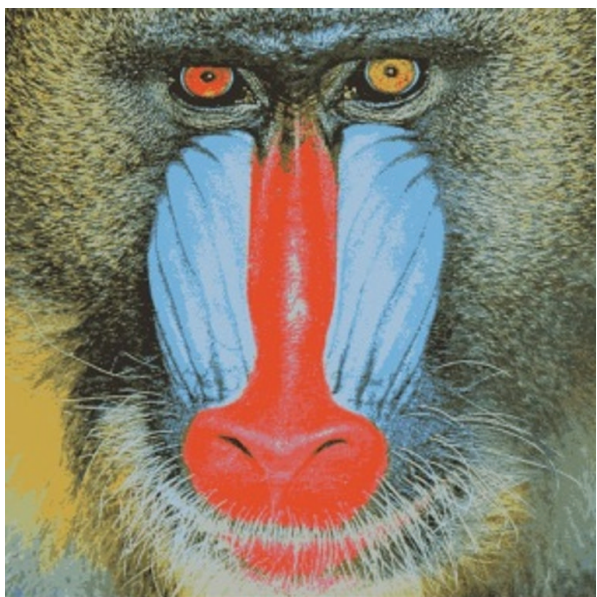

(c) SaDE-CIQ3

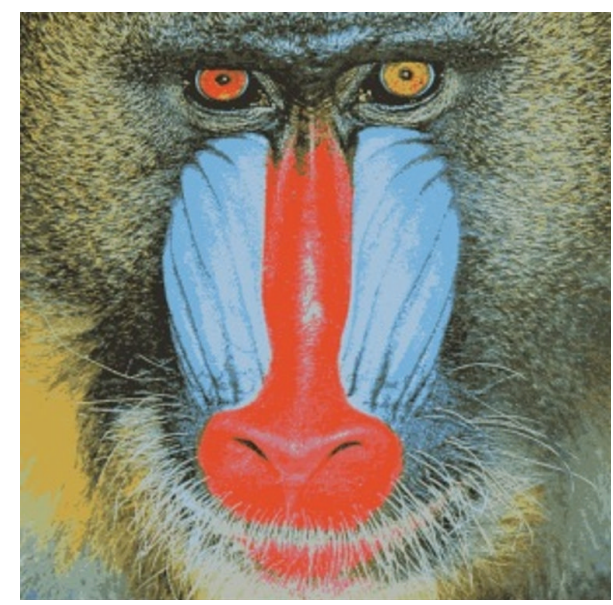

(b) MoDE-CIQ

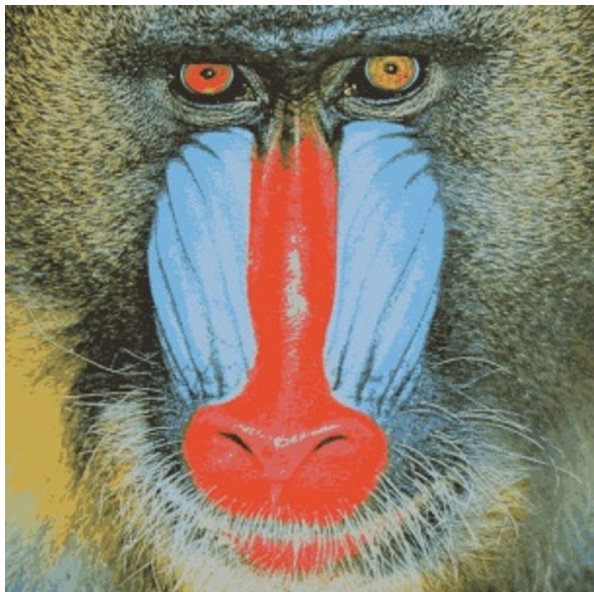

(d) SaDE-CIQ

Figure 4: The quantized images of Baboon with 16 colors obtained by three algorithms.

similar effects. (iii) For Lena (shown in Figure 5), there are many shaded regions in it. So differences in the quantization quality of the corresponding quantized images depend on the transition from shaded regions to highlights. MoDE-CIQ obtains the quantized image with more natural transition than SaDE-CIQ and SaDE-CIQ3. (iv) For Airplane (shown in Figure 6), there are extremely unequally distributed base colors. Obviously, the quantized image of SaDE-CIQ3 has the largest color distortion. Although the quantized image of SaDE-CIQ has a little better color distortion than that of the multiobjective algorithm, the former loses some detail colors, such as the cloud in the sky.

According the above results, for the images with contrasting and equally distributed main base colors, the quantization effects of MoDE-CIQ and SaDE-CIQ are similar. But for the images with many shaded regions and extremely unequally distributed base colors, MoDE-CIQ could make the colors more natural and preserve more detail colors. In SaDE-CIQ3, the weighted factors in (10) affect its quantization quality.
Thus, we can think MoDE-CIQ is superior to the other two algorithms.

4.2. Experiments for Showing the Advantage of the Multiobjective Model. As the statement on Step 4 of MoDE-CIQ, we can obtain an approximative Pareto solution set. This is an advantage comparing to all single-objective algorithms. The above experiments reserved the approximative Paretooptimal solutions of all four images. The solution sets corresponding to Peppers, Baboon, Lena, and Airplane, respectively, include 13 solutions (shown in Table 5), 9 solutions (in Table 6), 11 solutions (in Table 7), and 8 solutions (in Table 8). For comparing these optimal solutions, their corresponding MSE values are listed. Figure 7 shows the Pareto front of these Pareto-optimal solutions. These optimization solutions present some quantized images with different effects. Users can select the suitable quantized image according to their requirements for the color gradations and details. 


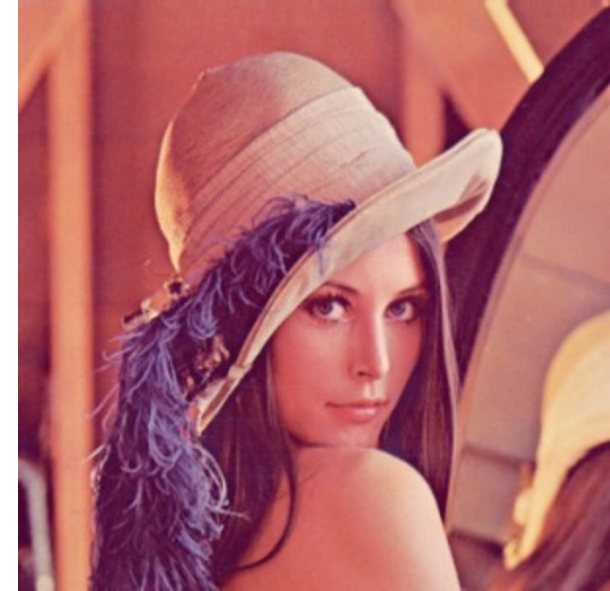

(a) Lena

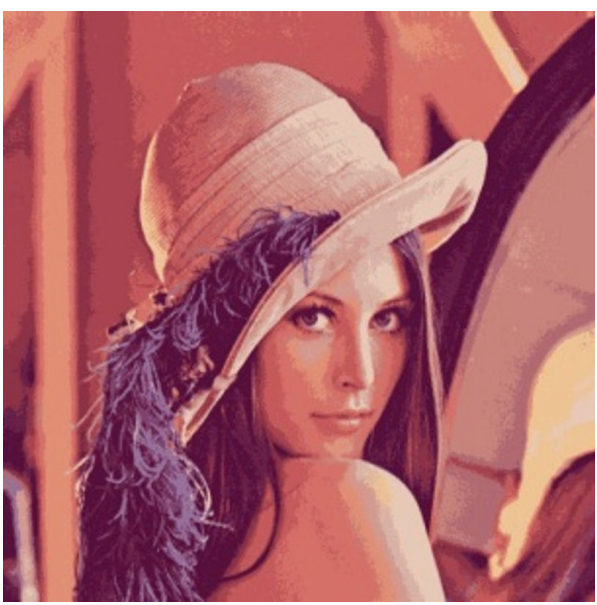

(c) SaDE-CIQ3

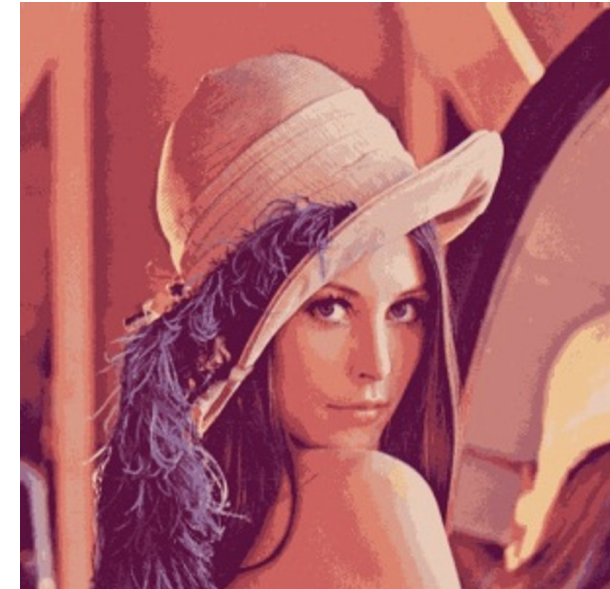

(b) MoDE-CIQ

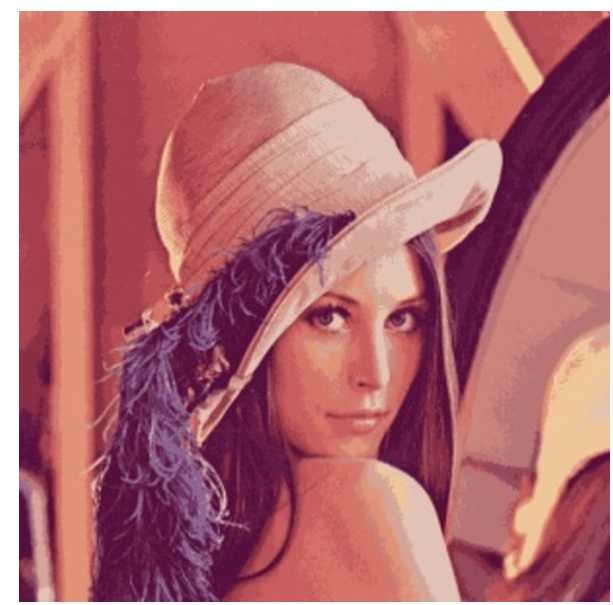

(d) SaDE-CIQ

FIGURE 5: The quantized images of Lena with 16 colors obtained by three algorithms.

TABle 5: Pareto-optimal solutions for Peppers.

\begin{tabular}{lccc}
\hline Order & $g_{1}(x)$ & $g_{2}(x)$ & MSE \\
\hline 1 & 24.9238 & 227.2425 & 19.5660 \\
2 & 38.5345 & 196.8913 & 25.4623 \\
3 & 31.0556 & 208.1886 & 24.1790 \\
4 & 34.6405 & 205.1429 & 22.2026 \\
5 & 25.6127 & 226.7033 & 19.1029 \\
6 & 35.2191 & 197.7366 & 23.0621 \\
7 & 31.4675 & 207.8684 & 23.3818 \\
8 & 34.4429 & 205.8758 & 22.2859 \\
9 & 34.8841 & 204.8451 & 26.031 \\
10 & 34.1102 & 207.7311 & 23.1158 \\
11 & 25.9563 & 217.8530 & 20.4536 \\
12 & 28.4238 & 210.6036 & 22.2533 \\
13 & 34.3636 & 206.7747 & 21.8853 \\
\hline
\end{tabular}

By the experimental results of the above two parts, MoDE-CIQ is a competitive algorithm for image color quantization.
TABle 6: Pareto-optimal solutions for Baboon.

\begin{tabular}{lccc}
\hline Order & $g_{1}(x)$ & $g_{2}(x)$ & MSE \\
\hline 1 & 27.3819 & 212.0693 & 23.1123 \\
2 & 27.8841 & 209.1716 & 22.9602 \\
3 & 31.8821 & 204.127 & 24.7008 \\
4 & 29.2681 & 205.8375 & 24.4271 \\
5 & 30.9412 & 204.9553 & 24.6433 \\
6 & 33.8514 & 202.2041 & 25.7050 \\
7 & 30.2455 & 205.5812 & 24.5084 \\
8 & 27.8998 & 208.0066 & 227.341 \\
9 & 27.6801 & 209.3535 & 227.341 \\
\hline
\end{tabular}

All the above algorithms were implemented in Visual $\mathrm{C}++$ and the experiments were conducted on a computer with Intel ${ }^{\circledR}$ Xeon ${ }^{\circledR}$ CPU E3-1230 v3 @ 3.30 GHZ and 8 GB RAM.

\section{Conclusions}

This paper established a multiobjective image color quantization model, in which intracluster distance $\bar{d}_{\max }$ and intercluster 


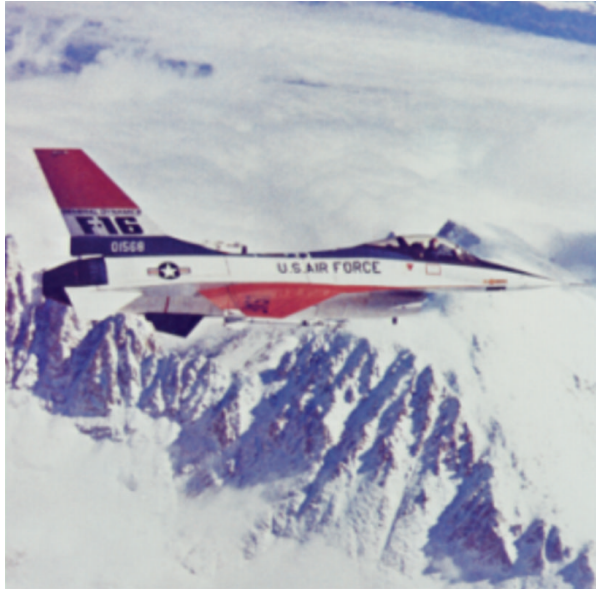

(a) Airplane

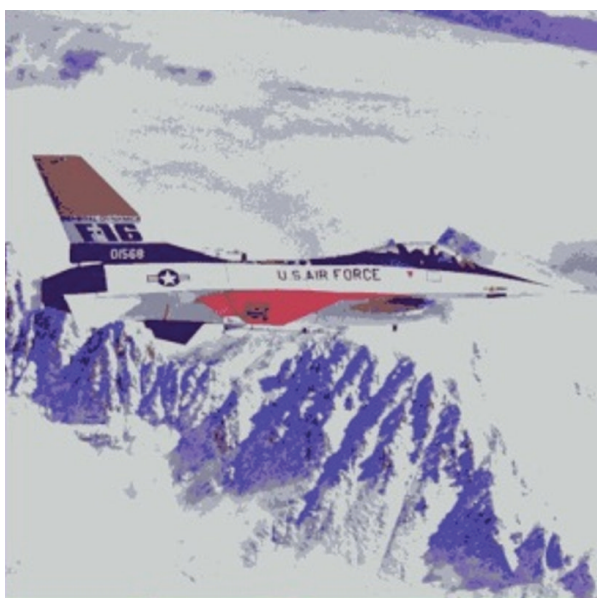

(c) SaDE-CIQ3

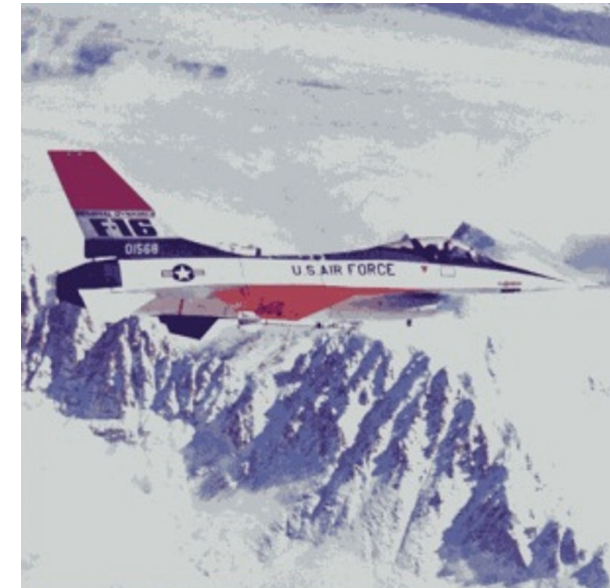

(b) MoDE-CIQ

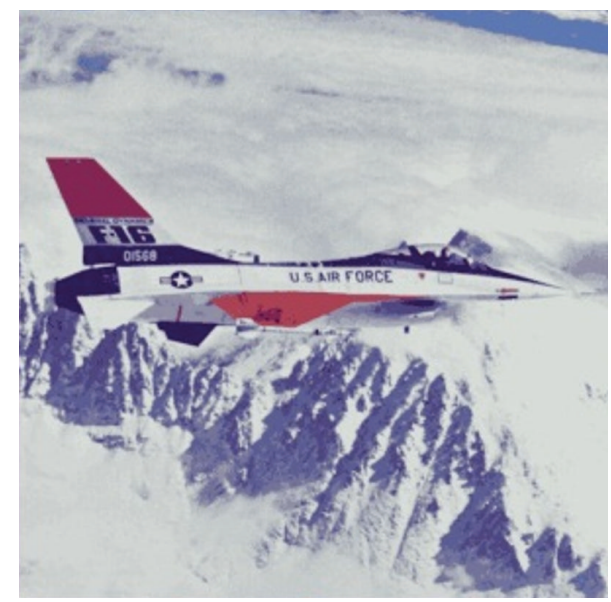

(d) SaDE-CIQ

FIgURE 6: The quantized images of Airplane with 16 colors obtained by three algorithms.

TABLE 7: Pareto-optimal solutions for Lena.

\begin{tabular}{lccc}
\hline Order & $g_{1}(x)$ & $g_{2}(x)$ & MSE \\
\hline 1 & 24.9313 & 207.9088 & 19.8533 \\
2 & 20.2311 & 228.5612 & 14.2847 \\
3 & 20.6109 & 228.4630 & 15.1880 \\
4 & 26.7185 & 202.3789 & 20.9155 \\
5 & 25.9452 & 203.7679 & 20.8445 \\
6 & 24.5586 & 209.2558 & 18.9967 \\
7 & 23.6997 & 209.7721 & 19.8327 \\
8 & 22.3126 & 212.7511 & 20.1288 \\
9 & 21.1493 & 216.7855 & 17.3328 \\
10 & 24.9279 & 209.1586 & 233.6600 \\
11 & 20.6396 & 224.5480 & 233.6600 \\
\hline
\end{tabular}

separation $d_{\min }$ are selected as its objective functions. A multiobjective image color quantization algorithm based on self-adaptive hybrid DE (MoDE-CIQ) was proposed to solve this model. MoDE-CIQ emerges the ideas of SaDE-CIQ [19]
TABLE 8: Pareto-optimal solutions for Airplane.

\begin{tabular}{lccc}
\hline Order & $g_{1}(x)$ & $g_{2}(x)$ & MSE \\
\hline 1 & 23.9419 & 220.4130 & 12.4973 \\
2 & 21.2536 & 225.4198 & 11.4192 \\
3 & 22.3128 & 223.4460 & 11.5732 \\
4 & 22.0105 & 225.3840 & 11.2520 \\
5 & 22.2876 & 225.0752 & 11.4864 \\
6 & 25.2011 & 219.2880 & 13.5785 \\
7 & 68.6311 & 212.3398 & 316.6950 \\
8 & 22.1871 & 225.3009 & 316.6950 \\
\hline
\end{tabular}

and a multipopulation DE algorithm VEDE [30], and applies MSE to determine the optimal solution. The multiobjective model and the proposed algorithm present a strategy to obtain a quantized image which holds the smallest color distortion among those images with a balance between the optimal color gradation and the optimal color details. The experimental results indicated that the multiobjective model 

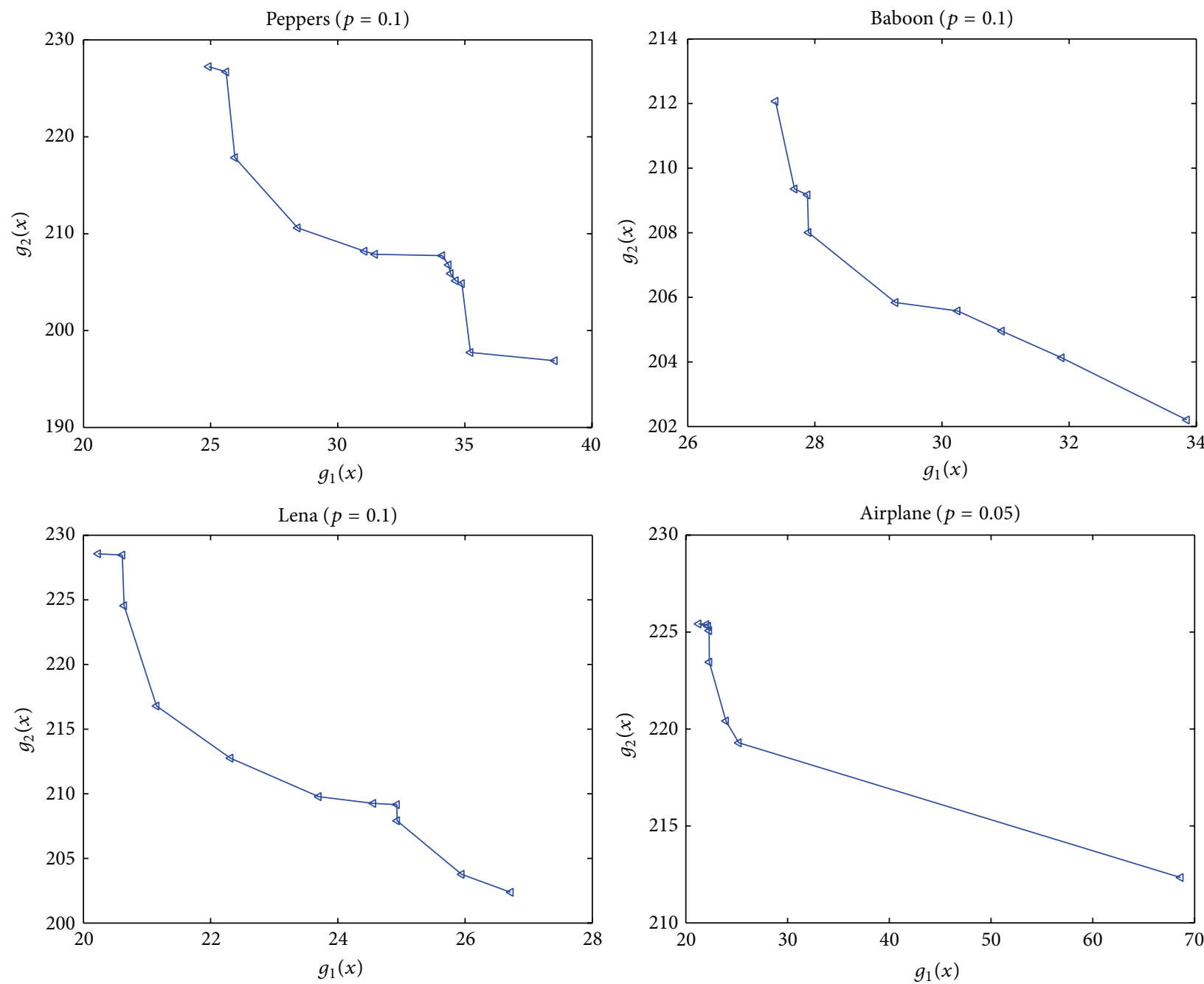

Figure 7: Pareto front of MoDE-CIQ.

and MoDE-CIQ are effective and competitive for image color quantization problems.

\section{Competing Interests}

The authors declare that there are no competing interests regarding the publication of this paper.

\section{Acknowledgments}

This work was supported in part by Hubei Province Department of Education Major Scientific Research Program of China (D20161306).

\section{References}

[1] J.-P. Braquelaire and L. Brun, "Comparison and optimization of methods of color image quantization," IEEE Transactions on Image Processing, vol. 6, no. 7, pp. 1048-1052, 1997.

[2] R. Kumar, A. Rohit, and P. Arup, "Color image quantization scheme using DBSCAN with K-means algorithm," in Intelligent
Computing, Networking, and Informatics: Proceedings of the International Conference on Advanced Computing, Networking, and Informatics, India, June 2013, vol. 243 of Advances in Intelligent Systems and Computing, pp. 1037-1045, Springer, Berlin, Germany, 2014.

[3] M. Emre Celebi, "Improving the performance of $k$-means for color quantization," Image and Vision Computing, vol. 29, no. 4, pp. 260-271, 2011.

[4] M. L. Pérez-Delgado, "Colour quantization with Ant-tree," Applied Soft Computing, vol. 36, pp. 656-669, 2015.

[5] X. D. Yue, D. Q. Miao, L. B. Cao, Q. Wu, and Y. F. Chen, "An efficient color quantization based on generic roughness measure," Pattern Recognition, vol. 47, no. 4, pp. 1777-1789, 2014.

[6] Y. Jiang, Y. Wang, L. Jin, H. Gao, and K. Zhang, "Investigation on color quantization algorithm of color image," in Advanced Research on Electronic Commerce, Web Application, and Communication, G. Shen and X. Huang, Eds., vol. 144 of Communications in Computer and Information Science, pp. 181187, Springer, Berlin, Germany, 2011.

[7] H. Palus and M. Frackiewicz, "New approach for initialization of K-means technique applied to color quantization," in Proceedings of the 2nd International Conference on Information 
Technology (ICIT '10), pp. 205-209, IEEE, Gdansk, Poland, June 2010.

[8] M. E. Celebi, "Fast color quantization using weighted sortmeans clustering," Journal of the Optical Society of America A, vol. 26, no. 11, pp. 2434-2443, 2009.

[9] G. Schaefer, Q. Hu, H. Zhou, J. F. Peters, and A. E. Hassanien, "Rough C-means and fuzzy rough C-means for colour quantisation," Fundamenta Informaticae, vol. 119, no. 1, pp. 113-120, 2012.

[10] E. J. Palomo and E. Domínguez, "Hierarchical color quantization based on self-organization," Journal of Mathematical Imaging and Vision, vol. 49, no. 1, pp. 1-19, 2014.

[11] H. J. Park, K. B. Kim, and E. Cha, "An effective color quantization method using octree-based self-organizing maps," Computational Intelligence and Neuroscience, vol. 2016, Article ID 5302957, 11 pages, 2016.

[12] M. E. Celebi, Q. Wen, and S. Hwang, "An effective realtime color quantization method based on divisive hierarchical clustering," Journal of Real-Time Image Processing, vol. 10, no. 2, article 291, pp. 329-344, 2015.

[13] P. Scheunders, "A genetic c-means clustering algorithm applied to color image quantization," Pattern Recognition, vol. 30, no. 6, pp. 859-866, 1997.

[14] T. Taşdizen, L. Akarun, and C. Ersoy, "Color quantization with genetic algorithms," Signal Processing: Image Communication, vol. 12, no. 1, pp. 49-57, 1998.

[15] M. G. Omran, A. P. Engelbrecht, and A. Salman, "A color image quantization algorithm based on Particle Swarm Optimization," Informatica, vol. 29, no. 3, pp. 261-269, 2005.

[16] J. Kennedy and W. M. Spears, "Matching algorithms to problems: an experimental test of the particle swarm and some genetic algorithms on the multimodal problem generator," in Proceedings of the IEEE International Conference on Evolutionary Computation (ICEC '98), pp. 78-83, IEEE, Anchorage, Alaska, USA, May 1998.

[17] K. Ryosuke, T. Hakaru, K. Hideaki et al., "A color quantization based on vector error diffusion and particle swarm optimization considering human visibility," in Image and Video Technology, vol. 9431, pp. 332-343, Springer, 2016.

[18] G. Schaefer and L. Nolle, "Optimal image colour extraction by differential evolution," International Journal of Bio-Inspired Computation, vol. 2, no. 3-4, pp. 251-257, 2010.

[19] Q. Su and Z. Hu, "Color image quantization algorithm based on self-adaptive differential Evolution," Computational Intelligence and Neuroscience, vol. 2013, Article ID 231916, 8 pages, 2013.

[20] C. Ozturk, E. Hancer, and D. Karaboga, "Color image quantization: a short review and an application with artificial bee colony algorithm," Informatica, vol. 25, no. 3, pp. 485-503, 2014.

[21] S. A. El-Said, "Image quantization using improved artificial fish swarm algorithm," Soft Computing, vol. 19, no. 9, pp. 2667-2679, 2015.

[22] S. Ouadfel, M. Batouche, and A. Taleb-Ahmed, "A modified particle swarm optimization algorithm for automatic image clustering," in Proceedings of the Symposium on Modelling and Implementation of Complex Systems (MISC '10), Constantine, Algeria, 2010.

[23] G. Schaefer, "Soft computing-based colour quantisation Soft Computing for Image Analysis," Eurasip Journal on Image and Video Processing, vol. 2014, article 8, 2014.

[24] M. E. Celebi, S. Hwang, and Q. Wen, "Colour quantisation using the adaptive distributing units algorithm," Imaging Science Journal, vol. 62, no. 2, pp. 80-91, 2014.
[25] M. Omran, A. Salman, and A. Engelbrecht, "Image classification using particle swarm optimization," in Proceedings of the 4th Asia-Pacific Conference on Simulated Evolution and Learning, Singapore, November 2002.

[26] M. Omran, A. P. Engelbrecht, and A. Salman, "Particle swarm optimization method for image clustering," International Journal of Pattern Recognition and Artificial Intelligence, vol. 19, no. 3, pp. 297-321, 2005.

[27] A. A. A. Esmin, D. L. Pereira, and F. P. A. de Araújo, "Study of different approach to clustering data by using the particle swarm optimization algorithm," in Proceedings of the IEEE Congress on Evolutionary Computation (CEC '08), pp. 1817-1822, IEEE, Hong Kong, June 2008.

[28] M. T. Wong, X. He, and W.-C. Yeh, "Image clustering using particle swarm optimization," in Proceedings of the IEEE Congress on Evolutionary Computation (CEC '11), pp. 262-268, New Orleans, La, USA, June 2011.

[29] G. Schaefer and L. Nolle, "A hybrid color quantization algorithm incorporating a human visual perception model," Computational Intelligence, vol. 31, no. 4, pp. 684-698, 2015.

[30] K. E. Parsopoulos, D. K. Tasoulis, N. G. Pavlidis, V. P. Plagianakos, and M. N. Vrahatis, "Vector evaluated differential evolution for multi-objective optimization," in Proceedings of the Congress on Evolutionary Computation (CEC '04), vol. 1, pp. 204-211, June 2004.

[31] M. G. H. Omran, A. P. Engelbrecht, and A. Salman, "Differential evolution methods for unsupervised image classification," in Proceedings of the IEEE Congress on Evolutionary Computation (CEC'05), vol. 2, pp. 966-973, Edinburgh, UK, September 2005. 

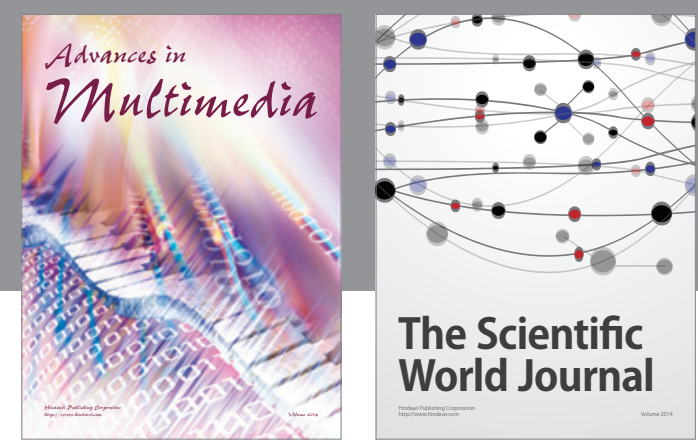

The Scientific World Journal
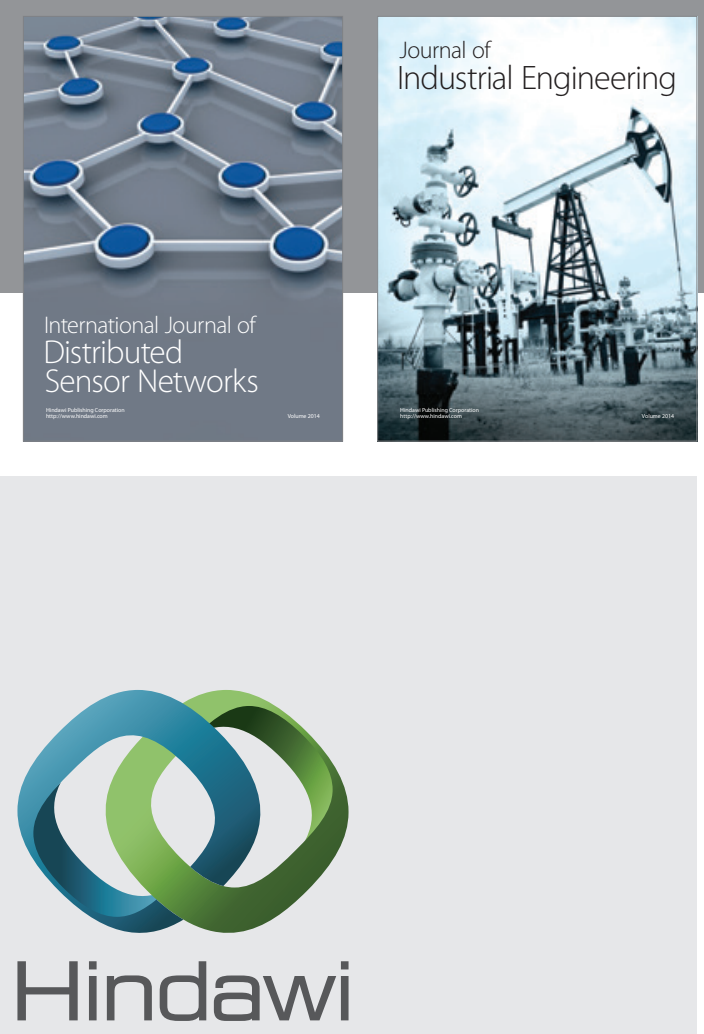

Submit your manuscripts at

http://www.hindawi.com

\section{Computer Networks} and Communications
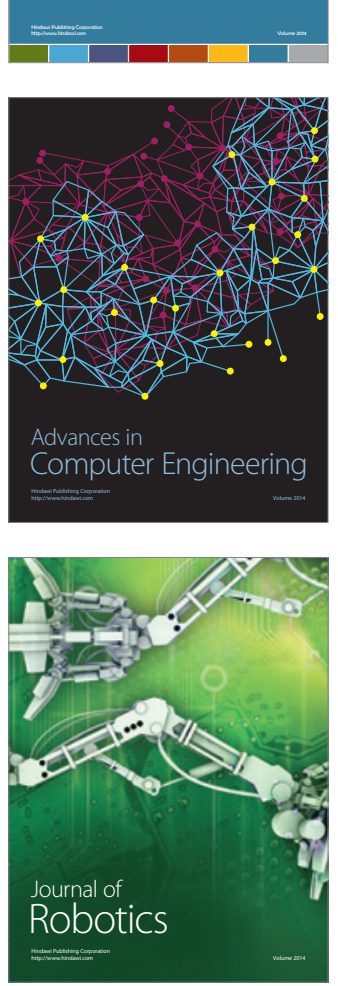
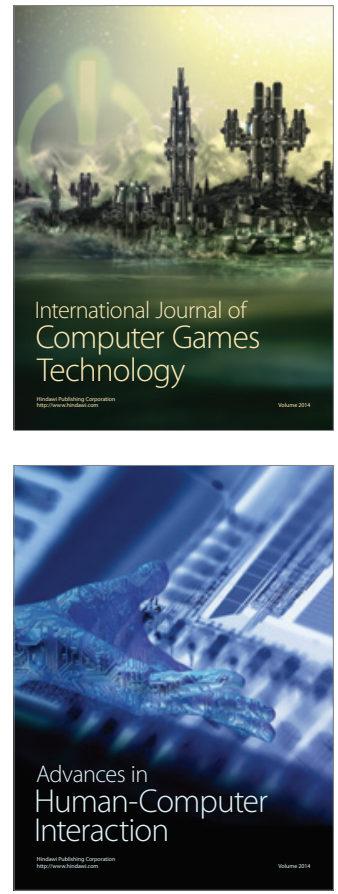
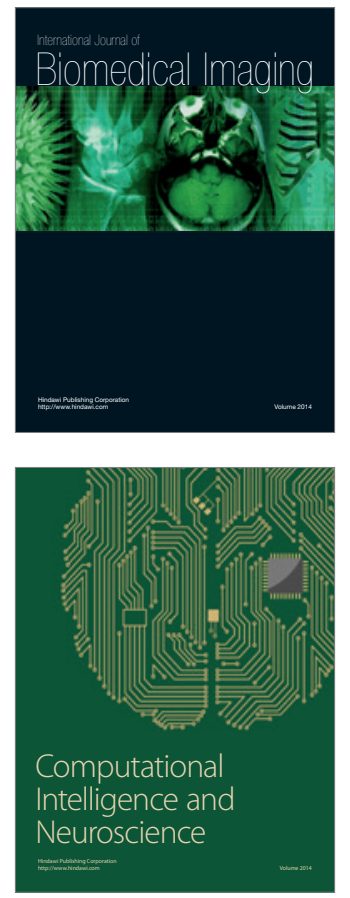
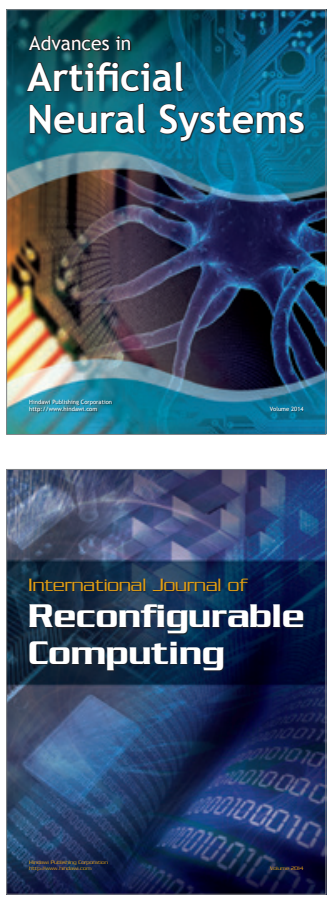
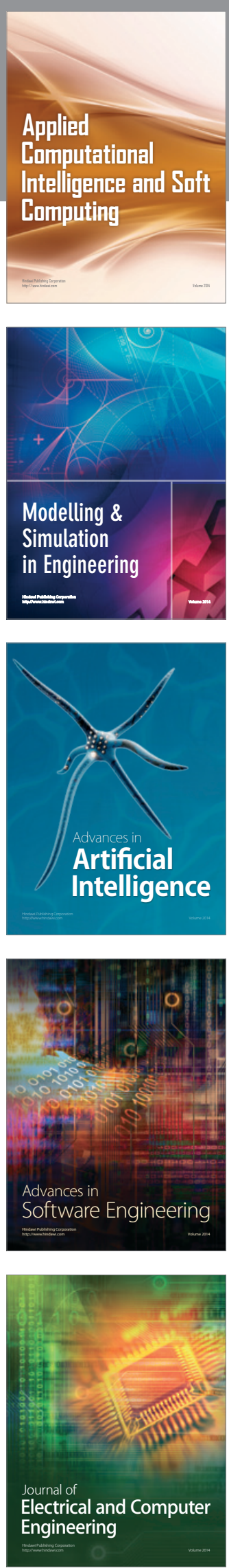